\title{
Extrinsic and Intrinsic Contributions to the Spin Hall Effect of Alloys
}

\author{
Stephan Lowitzer, ${ }^{1}$ Martin Gradhand, ${ }^{2,3}$ Diemo Ködderitzsch, ${ }^{1, *}$ Dmitry V. Fedorov, ${ }^{3}$ Ingrid Mertig, ${ }^{2,3}$ and Hubert Ebert ${ }^{1}$ \\ ${ }^{1}$ Department Chemie, Physikalische Chemie, Universität München, Butenandstr. 5-13, 81377 München, Germany \\ ${ }^{2}$ Max-Planck-Institut für Mikrostrukturphysik, Weinberg 2, D-06120 Halle, Germany \\ ${ }^{3}$ Institut für Physik, Martin-Luther-Universität Halle-Wittenberg, D-06099 Halle, Germany
}

(Received 27 October 2010; published 4 February 2011)

\begin{abstract}
A fully relativistic description of the spin-orbit induced spin Hall effect is presented that is based on Kubo's linear response formalism. Using an appropriate operator for the spin-current density a KuboStreda-like equation for the spin Hall conductivity (SHC) is obtained. An implementation using the Korringa-Kohn-Rostoker band structure method in combination with the coherent potential approximation allow detailed investigations on various alloy systems. A decomposition of the SHC into intrinsic and extrinsic contributions is suggested. Accompanying calculations for the skew-scattering contribution of the SHC using the Boltzmann equation demonstrate the equivalence to the Kubo formalism in the dilute alloy regime and support the suggested decomposition scheme.
\end{abstract}

DOI: 10.1103/PhysRevLett.106.056601

The emerging research field of spintronics has developed very rapidly during recent years. The reason for the broad interest in this field is based on the close connection to fundamental scientific questions as well as its impact on technology [1,2]. In this context, the spin Hall effect (SHE) is one of the most promising phenomena. It denotes the observation that a charge current flowing through a solid is accompanied by a transversal spin current. This occurs even for nonmagnetic solids as was demonstrated by experiments on pure $\mathrm{Pt}[3]$.

Both the anomalous Hall effect (AHE) in ferromagnets and the SHE are caused by the influence of spin-orbit coupling (SOC). Accordingly, their theoretical description is quite similar [4-15]. For ideal systems an intrinsic mechanism was identified which allows the expression of the corresponding response function in terms of the Berry curvature [5,7]. On this basis, ab initio calculations for the intrinsic spin Hall conductivity (SHC) were performed [8-11]. As for the AHE, the additional extrinsic SHC in dilute and concentrated alloys is ascribed to skew and sidejump scattering caused by SOC. The role of these mechanisms for the SHE has been studied so far primarily by model calculations $[12,13]$. First principle calculations for the extrinsic SHC of dilute alloys on the basis of the Boltzmann formalism that account for the skew-scattering mechanism have been performed only very recently $[14,15]$. However, a complete description of intrinsic and extrinsic mechanisms giving rise to the SHE applicable to ideal as well as alloy systems, as it is presented below, was missing so far. As pointed out by several authors [16,17], a central issue for such an approach is an adequate definition for the spin-current density operator that accounts for SOC. This was supplied recently by Vernes et al. [18] by starting from the Bargmann-Wigner four-vector spin polarization operator $\mathcal{T}$ [19]. Demanding that the spin polarization is connected with the spin-current density
PACS numbers: 72.25.Ba, 71.15.Rf, 75.76.+j, 85.75.-d

via a corresponding continuity equation an explicit expression for the spin-current operator was given.

An adequate formal basis for the discussion of the SHE in nonmagnetic metals is supplied by Kubo's linear response formalism that allows the derivation of an expression for the spin Hall conductivity tensor. To avoid any approximation when dealing with SOC the underlying electronic structure is described in a fully relativistic way by the four-component Dirac formalism [20]. As for the ordinary electrical conductivity the perturbation due to the external electric field is represented in terms of the current density operator $\hat{\mathbf{j}}$. In its relativistic form this operator is given by

$$
\hat{\mathbf{j}}=-|e| c \boldsymbol{\alpha},
$$

where $\boldsymbol{\alpha}$ is the vector-matrix of the standard Dirac matrices $\alpha_{i}[20]$ and the other quantities have their usual meaning. The response function to be considered for the SHE is the spin-current density. Considering for the $z$ component of the spin polarization vector the current density along the $x$ direction the corresponding operator is given by $[18,21,22]$ :

$$
\hat{J}_{x}^{z}=|e| c \alpha_{x}\left(\beta \Sigma_{z}-\frac{1}{m c} \gamma_{5} \hat{p}_{z}\right),
$$

where $\hat{p}_{z}$ is the canonical momentum operator, $\Sigma_{z}$ is the $z$ component of the vector of the relativistic spin matrix, $\beta$ and $\gamma_{5}$ are Dirac matrices [20].

Adopting a single-particle description of the electronic structure in terms of the retarded $\left(G^{+}\right)$and advanced $\left(G^{-}\right)$ Green's function and restricting to $T=0 \mathrm{~K}$, an explicit expression for the SHC is obtained that is similar to the Kubo-Středa equation for the anomalous Hall conductivity $\sigma_{x y}$ of ferromagnetic systems. Considering for the spin polarization along $\hat{z}$ its current density along $\hat{x}$ due to an electric field along $\hat{y}$, the SHC $\sigma_{x y}^{z}$ is given by [23]: 


$$
\begin{aligned}
\sigma_{x y}^{z}= & \frac{\hbar}{2 \pi N \Omega} \operatorname{Tr}\left\langle\hat{J}_{x}^{z} G^{+} \hat{j}_{y} G^{-}\right\rangle_{c} \\
& +\frac{|e|}{4 \pi i N \Omega} \operatorname{Tr}\left\langle\left(G^{+}-G^{-}\right)\left(\hat{r}_{x} \hat{J}_{y}^{z}-\hat{r}_{y} \hat{J}_{x}^{z}\right)\right\rangle_{c},
\end{aligned}
$$

where terms containing products of the retarded (or advanced) Green's functions have been dropped [6]. Because of symmetry the last term is site-diagonal for the cubic systems considered here. As furthermore all system considered here are metallic [12,24] it has been omitted as well.

The electronic Green's function occurring in Eq. (3) for the Fermi energy $E_{F}$ can be evaluated in a very efficient way by use of the relativistic version of the multiple scattering or Korringa-Kohn-Rostoker (KKR) formalism [25]. This approach is applicable to ideal systems but can also be applied straightforwardly to disordered alloys. For this case the brackets $\langle\ldots\rangle_{c}$ in Eq. (3) imply a configurational average that is performed within the coherent potential approximation (CPA) [26]. For this purpose the KKR-CPA approach used for the electrical conductivity tensor of alloys on the basis of the Kubo-Středa equation [27] has been adapted to Eq. (3). In particular, this approach accounts explicitly for the so-called vertex corrections which represent the difference in the correlated and uncorrelated configurational averages of the type $\left\langle\hat{J}_{x}^{z} G^{+} \hat{j}_{y} G^{-}\right\rangle_{c}$ and $\left\langle\hat{J}_{x}^{z} G^{+}\right\rangle_{c}\left\langle\hat{j}_{y} G^{-}\right\rangle_{c}$, respectively. For the following it is important to note that the vertex corrections correspond to the scattering-in term within the Boltzmann formalism [26].

Representing the anomalous Hall conductivity (AHC) $\sigma_{x y}$ in terms of Feynman diagrams it was demonstrated that all extrinsic contributions to $\sigma_{x y}$ due to skew and sidejump scattering correspond to terms involving vertex corrections [28]. Obviously, the same conclusion can be drawn for the SHC $\sigma_{x y}^{z}$. The remaining diagrams, involving no vertex corrections, stand for the intrinsic anomalous or spin Hall conductivity, plus corrections to this due to chemical disorder. It therefore seems natural to extend the definition of the intrinsic SHC $\sigma_{x y}^{z \text { intr }}$ to diluted and concentrated alloys to represent all contributions not connected to the vertex corrections, as has already been hinted at in Refs. [6,29] According to this definition, Eq. (3), including the vertex corrections, gives the total SHC $\sigma_{x y}^{z} \equiv$ $\sigma_{x y}^{z \mathrm{VC}}$ while the intrinsic SHC $\sigma_{x y}^{z \text { intr }} \equiv \sigma_{x y}^{z \text { noVC }}$ is obtained if those are ignored. Thus, $\sigma_{x y}^{z \text { intr }}$ is the intrinsic SHC of the effective CPA medium specific for each composition of a certain alloy. As a consequence, the extrinsic SHC $\sigma_{x y}^{z \text { extr }}$ to be ascribed to the skew and side-jump scattering mechanisms is obtained from the difference $\sigma_{x y}^{z \text { extr }}=$ $\sigma_{x y}^{z}-\sigma_{x y}^{z \text { intr }}$.

The approach sketched above has been applied to investigate the SHE for the fcc alloy systems $\mathrm{Au}_{x} \mathrm{Pt}_{1-x}$ and $\mathrm{Ag}_{x} \mathrm{Au}_{1-x}$. As can be seen from Fig. $1 \sigma_{x y}^{z \text { intr }}$ obtained from Eq. (3) ignoring the vertex corrections varies nearly linearly with the concentration throughout the whole
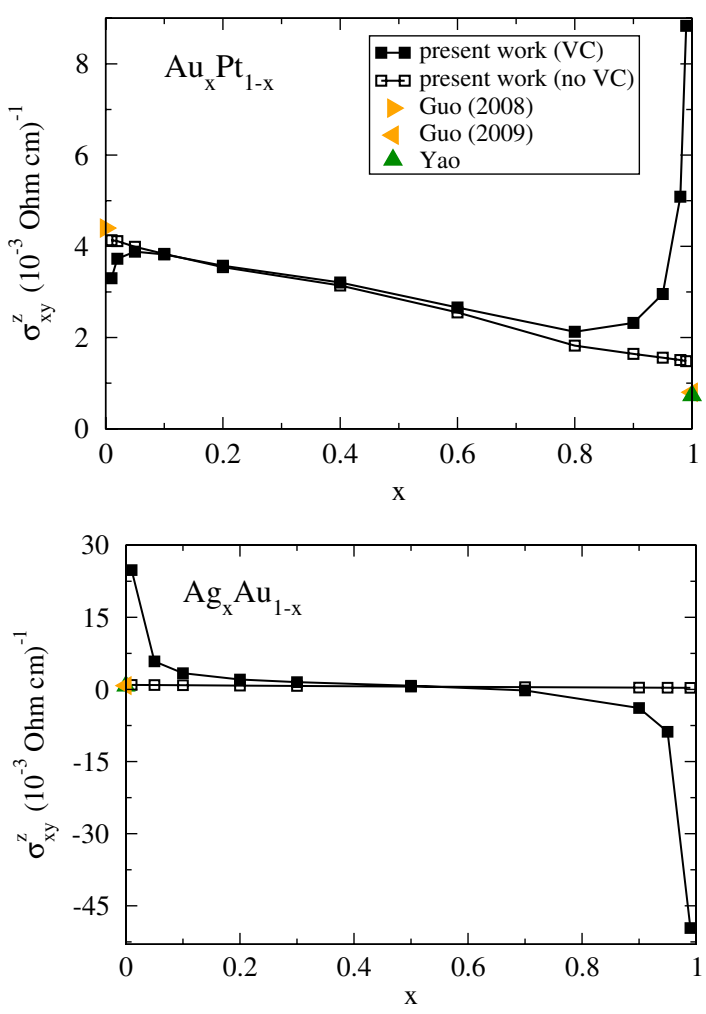

FIG. 1 (color online). Spin Hall conductivity $\sigma_{x y}^{z}$ for the alloy systems $\mathrm{Au}_{x} \mathrm{Pt}_{1-x}$ and $\mathrm{Ag}_{x} \mathrm{Au}_{1-x}$. The full squares correspond to calculations including vertex corrections while the open squares represent calculations without vertex corrections. Results from other $a b$ initio investigations on the intrinsic SHE of pure Pt [10] and $\mathrm{Au}[8,11]$ are included. Because of the different definitions for the spin-current operator the results from Refs. [10,11] have been multiplied by a factor of 2 for the sake of consistency.

composition regime. In addition, Fig. 1 (top) shows results for the intrinsic SHC of $\mathrm{Au}[8,11]$ and $\mathrm{Pt}[10]$ obtained by other authors using an expression for $\sigma_{x y}^{z \text { intr }}$ in terms of the Berry curvature. Taking into account the differences between the various calculation schemes used-in particular concerning the treatment of spin-orbit coupling and the definition of the spin-current density operator-these data fit reasonably well with the alloy data obtained using the Kubo-Středa-like equation [Eq. (3)]. This obviously justifies the extension of the definition for $\sigma_{x y}^{z \text { intr }}$ to the alloy case to represent all terms that do not involve the vertex corrections.

In contrast to the intrinsic SHC, the total one $\left(\sigma_{x y}^{z}\right)$ shows for both investigated alloy systems a divergent behavior in the dilute limit when the concentration $x$ approaches 0 or 1 , respectively. Interestingly, the corresponding extrinsic SHC $\sigma_{x y}^{z \text { extr }}$ changes sign when the concentration $x$ varies from 0 to 1 . For concentrated alloys $(0.2<x<0.8)$ the intrinsic and total SHC do not differ strongly. As this behavior is also found for other alloy systems that do not show a change in sign for the extrinsic SHC $\sigma_{x y}^{z \text { extr }}$ it seems that the impact of the vertex corrections in the concentrated alloy regime is in general negligible. 
For the anomalous Hall effect it is known that one may classify solid state materials according to the scaling relation between the AHC $\sigma_{x y}$ and the longitudinal conductivity $\sigma_{x x}$. For metallic systems those fall in general into the so-called ultraclean regime $\left(\sigma_{x x} \gtrsim 10^{6}(\Omega \mathrm{cm})^{-1}\right)$ the skew-scattering mechanism should dominate $\sigma_{x y}[30,31]$. In this case the scaling relation $\sigma_{x y} \approx \sigma_{x y}^{\text {skew }}=S \sigma_{x x}$ holds with $S$ the so-called skewness factor. Assuming the same to apply for the SHE as well, one may expect for the extrinsic SHC the relation:

$$
\sigma_{x y}^{z \text { extr }}=\sigma_{x y}^{z \text { skew }}+\sigma_{x y}^{z \text { sj }}=S^{z} \sigma_{x x}+\sigma_{x y}^{z \mathrm{sj}}
$$

where $S^{z}$ is the corresponding skewness factor and the term $\sigma_{x y}^{z \text { sj }}$ represents the contribution due to the side-jump mechanism.

Plotting the extrinsic SHC $\sigma_{x y}^{z e x t r}$ of $\mathrm{Au}_{x} \mathrm{Pt}_{1-x}$ and $\mathrm{Ag}_{x} \mathrm{Au}_{1-x}$ versus the corresponding $\sigma_{x x}$ with the concentration as an implicit parameter indeed a linear behavior is found in the dilute regimes ( $x \leq 0.1$ or $x \geq 0.9)$, as can be seen from Fig. 2. Fitting a straight line to the data for the considered systems and extrapolating to $\sigma_{x x}=0$ allows us to deduce the side-jump contribution on the basis of Eq. (4). The results obtained for $\mathrm{Au}_{x} \mathrm{Pt}_{1-x}$ and $\mathrm{Ag}_{x} \mathrm{Au}_{1-x}$ are shown in Fig. 3 together with data obtained for two
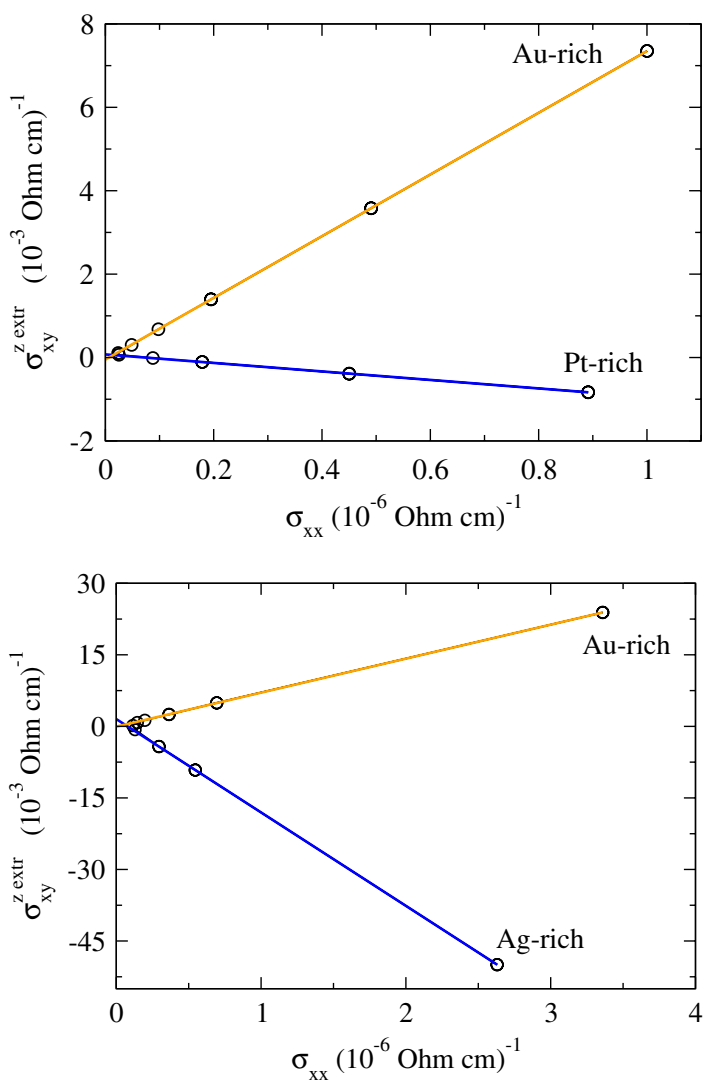

FIG. 2 (color online). The extrinsic spin Hall conductivity $\sigma_{x y}^{z \text { extr }}$ versus the longitudinal conductivity $\sigma_{x x}$ for $\operatorname{Ag}_{x} \mathrm{Au}_{1-x}$ and $\mathrm{Au}_{x} \mathrm{Pt}_{1-x}$ (black line/circles). The blue and orange lines are explained in the text. other alloy systems. Obviously, $\sigma_{x y}^{z \text { sj }}$ may take either sign and is in most cases found to be much smaller than the intrinsic contribution. On the other hand, $\sigma_{x y}^{z \text { skew }}$ is dominating in the dilute limit since it scales with the concentration, while $\sigma_{x y}^{z \text { intr }}$ does not depend on the concentration explicitly. However, an actual impurity concentration for the crossover between intrinsic and skew-scattering regime depends on the considered alloy.

To support the analysis of the results for the total SHC presented above, complementary work has been done using the Boltzmann formalism for the SHE [14]. The results for $\sigma_{x x}$ obtained this way are found in very good agreement with those obtained using the Kubo-Greenwood equation (see top panel of Fig. 4). As mentioned above, the vertex corrections giving rise to $\sigma_{x y}^{z}$ extr correspond to the scattering-in processes occurring in the Boltzmann formalism. As it was demonstrated recently, the latter ones give rise to the skew-scattering mechanism [14]. Corresponding results for $\sigma_{x y}^{z \text { skew }}$ (bottom panel of Fig. 4) are also found in very satisfying agreement with the results based on the Kubo-Středa-like formula [Eq. (3)] together with the described decomposition. This finding convincingly shows the equivalence of both approaches for the dilute alloy regime and it also justifies once more the used definition for the intrinsic SHC introduced above.

In summary, an expression for the spin Hall conductivity $\sigma_{x y}^{z}$ has been derived in analogy to the Kubo-Streda equation for the anomalous Hall conductivity $\sigma_{x y}$ of ferromagnets. An implementation within the fully relativistic KKR-CPA formalism allows material specific $a b$ initio investigations for various transition metal alloy systems over the whole range of composition dealing with all contributions to the SHC on the same footing. We decompose the total SHC $\sigma_{x y}^{z}$ into its intrinsic and extrinsic parts. In the concentrated alloys the intrinsic contribution of the effective medium always dominates. The extrinsic contribution, on the other hand, shows in general a diverging behavior in the dilute alloy regime that is ascribed to the

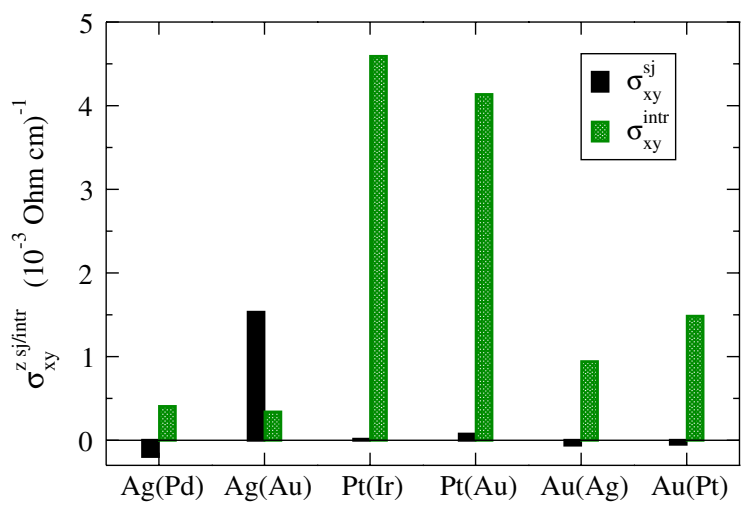

FIG. 3 (color online). The side-jump and intrinsic contributions to the spin Hall conductivity, $\sigma_{x y}^{z \text { sj }}$ and $\sigma_{x y}^{z \text { intr }}$, respectively, for various dilute transition metal alloys $A(B)$ with the concentration of 1 at. \% for the dissolved component $B$. 

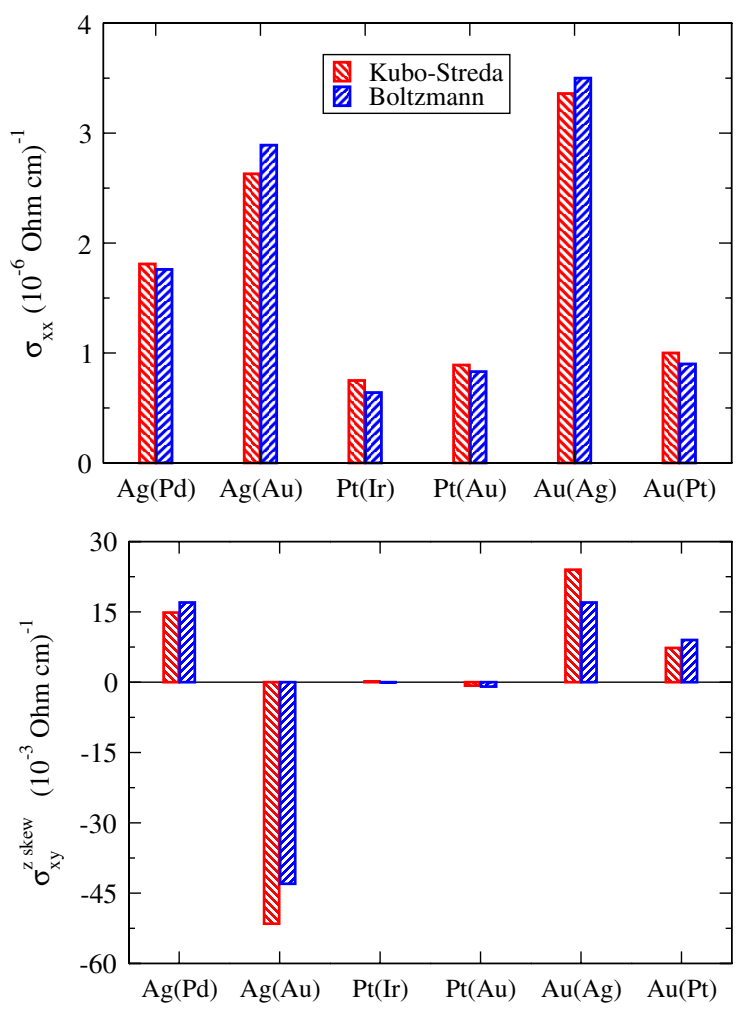

FIG. 4 (color online). The longitudinal conductivity $\sigma_{x x}$ (top) and the skew-scattering contribution to the spin Hall conductivity $\sigma_{x y}^{z \text { skew }}$ (bottom) for various dilute alloys $A(B)$ obtained on the basis of the Kubo-Středa equation (left bar) and the Boltzmann formalism (right bar), respectively. The concentration for the dissolved component $B$ is 1 at. \%.

skew-scattering contribution. Accompanying calculations on the basis of the Boltzmann formalism demonstrate its equivalence with the Kubo formalism in the dilute alloy regime and support the decomposition made for the total SHC $\sigma_{x y}^{z}$. At the moment clear-cut quantitative statements concerning the composition of the samples are lacking in experiment. In particular, for the spin-Hall-angle for $\mathrm{Au}$ and Pt several different experimental values are reported. From our study it is clear that especially in the dilute limit the SHC shows a sensitive concentration dependence. Experimental work is therefore needed to obtain SHC data from well characterized samples over a broad concentration range to clearly see the alloy behavior found in this work.

The authors S. L., D. K., and H.E. would like to thank the DFG for financial support within the SFB 689 "Spinphänomene in reduzierten Dimensionen." Furthermore, this work was supported by the International Max Planck Research School for Science and Technology.

*dkopc@cup.uni-muenchen.de

[1] S. A. Wolf, D. D. Awschalom, R. A. Buhrman, J. M. Daughton, S. von Molnár, M. L. Roukes, A. Y.
Chtchelkanova, and D.M. Treger, Science 294, 1488 (2001).

[2] D. Awschalom and N. Samarth, Physics 2, 50 (2009).

[3] T. Kimura, Y. Otani, T. Sato, S. Takahashi, and S. Maekawa, Phys. Rev. Lett. 98, 156601 (2007).

[4] A. Fert, A. Friedrich, and A. Hamzic, J. Magn. Magn. Mater. 24, 231 (1981).

[5] T. Jungwirth, Q. Niu, and A. H. MacDonald, Phys. Rev. Lett. 88, 207208 (2002).

[6] N. Nagaosa, J. Sinova, S. Onoda, A. H. MacDonald, and N. P. Ong, Rev. Mod. Phys. 82, 1539 (2010).

[7] J. Sinova, D. Culcer, Q. Niu, N. A. Sinitsyn, T. Jungwirth, and A. H. MacDonald, Phys. Rev. Lett. 92, 126603 (2004).

[8] Y. Yao and Z. Fang, Phys. Rev. Lett. 95, 156601 (2005).

[9] G. Y. Guo, Y. Yao, and Q. Niu, Phys. Rev. Lett. 94, 226601 (2005).

[10] G. Y. Guo, S. Murakami, T.-W. Chen, and N. Nagaosa, Phys. Rev. Lett. 100, 096401 (2008).

[11] G. Y. Guo, J. Appl. Phys. 105, 07 C701 (2009).

[12] T. Tanaka, H. Kontani, M. Naito, T. Naito, D. S. Hirashima, K. Yamada, and J. Inoue, Phys. Rev. B 77, 165117 (2008).

[13] G. Y. Guo, S. Maekawa, and N. Nagaosa, Phys. Rev. Lett. 102, 036401 (2009).

[14] M. Gradhand, D. V. Fedorov, P. Zahn, and I. Mertig, Phys. Rev. Lett. 104, 186403 (2010).

[15] M. Gradhand, D. V. Fedorov, P. Zahn, and I. Mertig, Phys. Rev. B 81, 245109 (2010).

[16] Q.-f. Sun and X. C. Xie, Phys. Rev. B 72, 245305 (2005).

[17] J. Shi, P. Zhang, D. Xiao, and Q. Niu, Phys. Rev. Lett. 96, 076604 (2006).

[18] A. Vernes, B. L. Györffy, and P. Weinberger, Phys. Rev. B 76, 012408 (2007).

[19] V. Bargmann and E. P. Wigner, Proc. Natl. Acad. Sci. U.S.A. 34, 211 (1948).

[20] M.E. Rose, Relativistic Electron Theory (Wiley, New York, 1961).

[21] S. Lowitzer, D. Ködderitzsch, and H. Ebert, Phys. Rev. B 82, 140402 (2010).

[22] Note, that different conventions are used in the literature for the spin-current. For the sake of comparison with literature we construct $\hat{J}_{x}^{z}$ such that it is given in units of charge conductivity. In addition, it has to be mentioned that the spin current was defined with opposite sign in previous publications by some of the authors [14,15].

[23] S. Lowitzer, Ph.D. thesis, LMU München, 2010.

[24] T. Naito, D. S. Hirashima, and H. Kontani, Phys. Rev. B 81, 195111 (2010).

[25] H. Ebert, in Electronic Structure and Physical Properties of Solids, edited by H. Dreyssé, Lecture Notes in Physics Vol. 535 (Springer, Berlin, 2000), p. 191.

[26] W. H. Butler, Phys. Rev. B 31, 3260 (1985).

[27] S. Lowitzer, D. Ködderitzsch, and H. Ebert, Phys. Rev. Lett. 105, 266604 (2010).

[28] A. Crépieux and P. Bruno, Phys. Rev. B 64, 014416 (2001).

[29] N. A. Sinitsyn, J. Phys. Condens. Matter 20, 023201 (2008).

[30] S. Onoda, N. Sugimoto, and N. Nagaosa, Phys. Rev. Lett. 97, 126602 (2006).

[31] S. Onoda, N. Sugimoto, and N. Nagaosa, Phys. Rev. B 77, 165103 (2008). 